\title{
RAPID ARCH EXPANSION IN CLEFT LIP AND PALATE CHILDREN: COMPARISON BETWEEN FAN-SHAPED EXPANDER AND HYRAX-TYPE EXPANDER BY USING CONE BEAM COMPUTED TOMOGRAPHY
}

\author{
Ahmed M. M. Sebaey, * Samy Youssef El-Bayomy, ${ }^{* *}$ \\ Youssef Hamed Abo Khalefa*** and Noha Ibrahim Abdel-Rahman ${ }^{* * * *}$
}

\begin{abstract}
Purpose: The aim of the current study was to evaluate the skeletal and dentoalveolar effects of rapid palatal expansion (RPE) in children with cleft lip and palate (CLP), ages 8 to 12 years treated with 2 expanders. Methods: Twenty Egyptian children with complete bilateral CLP exhibiting maxillary transverse deficiency were divided randomly into 2 groups according to the appliance used for expansion: (A) fan expander group. (B) hyrax expander group. Cone-beam computed tomography (CBCT) images were taken before and immediately after expansion procedure. Results: There was non-significant vertical movement of the maxilla for the 2 groups. On the transverse plane, there was more anterior expansion and posterior restriction for the fan group. However, the hyrax group showed significant posterior as well as anterior expansion. Both groups showed significant tipping between both sides posteriorly and anteriorly. Mandibular expansion was non-significant. Conclusion: Fan expander showed better results for children with cleft palate requiring anterior expansion only. Hyrax expander is better used for cases seeking for posterior and anterior expansion.
\end{abstract}

\section{INTRODUCTION}

One of the most prevalent birth defects affecting children around the world is the cleft lip and/or palate (CLP), where it is estimated to affect the quality of life of 7 million people worldwide.,
Surgical repairs of the lip and palate in the first few years of life during the development of the maxillary arch, might result in a narrow palatal vault. Compromised by scar tissues, along with absence of the mid-palatine suture, anterior and/

\footnotetext{
* Faculty of Dental Medicine, Al-Azhar University (Assiut Branch), Dentist at Egypt Ministry of Health.

** Professor and Head, Department of Pedodontics and Dental Public Health Faculty of Dental Medicine, Boys, Cairo, Al-Azhar University.

*** Lecturer, Department of Pedodontics and Dental Public Health Faculty of Dental Medicine, Boys, Cairo, Al-Azhar University. ***** Lecturer, Department of Orthodontics, Faculty of Dentistry, Ain Shams University.
} 
or posterior maxillary constriction is usually the consequence., Subsequently, rapid palatal expansion (RPE) is a common orthopedic procedure to correct this transverse deficiency, usually carried out during the mixed dentition phase aiming to widen the arch before alveolar bone graft surgery to substitute missing bone in the cleft alveolus. The variability of the cleft defect, and its impact on the surrounding anatomy, the effects of RPE in children with CLP seem to be different than in children without this deformity. ${ }^{(6-12)}$

Various expander designs are being used to cater to the anatomic variability present in the clefted maxilla ${ }^{(13)}$; the infamous Hyrax, and the Fan-shaped expander are among the most common expanders we use in our center. Consequently, our aim was to study radiographically the three dimensional skeletal and dentoalveolar effects of these two expanders with the aid of the cone beam computed tomography (CBCT), known for its lower radiation emission than regular $\mathrm{CT}$, in addition to its high resolution and accurate $3 \mathrm{D}$ representation of the imaged structure. ${ }^{(14)}$

\section{PARTICIPANTS AND METHODS}

Initially, 26 Egyptian children with complete bilateral CLP, ranging in age from 8 to 12 years (mean $=10$ years) selected from the outpatient clinic of the Orthodontic Department, the Cleft Care Center affiliated to the Oral and Maxillofacial Surgery Department, Faculty of Dentistry- Ain Shams University, and the outpatient clinic of Oral and Maxillofacial Surgery Department- Sayed Galal Hospital. All the children had transverse anterior and or posterior maxillary deficiency, requiring palatal expansion prior to alveolar bone grafting surgery. Children with syndromic cleft lip and palate, bad oral hygiene, or who had undergone previous orthopedic expansion were excluded from the study. ${ }^{(15)}$ Two children parents' inability to fulfill the required visits due to the long travelling distance, resulted in their children's removal from the study. One child had difficulty complying to the expansion protocol, therefore also dropping out resulting in a total of 23 participants. Children with no crossbite at the first permanent molars were assigned to group A, the Fan-shaped* expander group (6 girls, 5 boys), while those with posterior crossbite were added to the Hyrax* group B, (5 girls, 7 boys). Both appliances were tooth borne, banded on the first permanent molars with st.st. extension arms extended anteriorly to the most anterior tooth in transverse crossbite, which in most cases was the deciduous canine. Fig 1,2. This was to standardize the appliance design and facilitate seating, which could have been challenging due to the malignment and missing teeth observed in most dental arches with the children in the study. Both appliances were fabricated in the same laboratory by the same technician.

Parents were instructed to turn the screw twice daily yielding $0.5 \mathrm{~mm}$ expansion per day. All the children were scanned at two different time points: $\mathbf{T 1}$, before the delivery of the expander and T2,

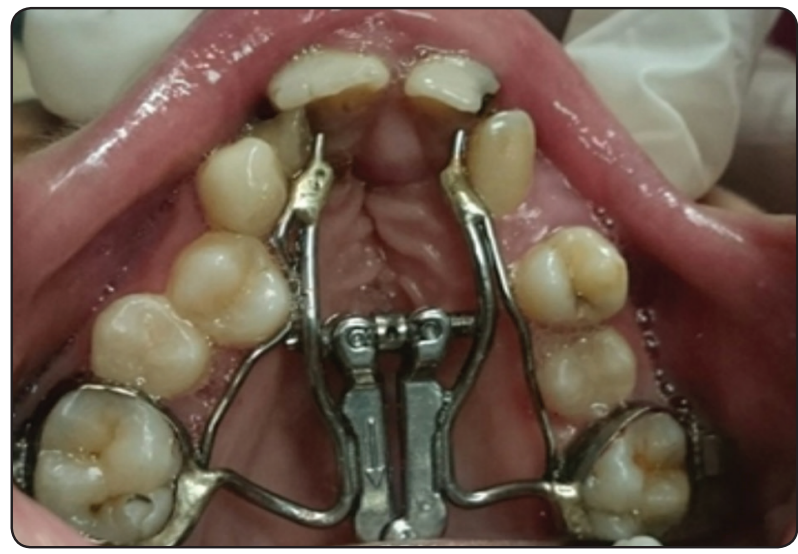

Fig. (1) Occlusal view of the banded fan-shaped expander, with extension arms reaching to the most anterior tooth in crossbite.

\footnotetext{
* Leone s.p.a. Ortodonzia e implantologia, Italy.
} 


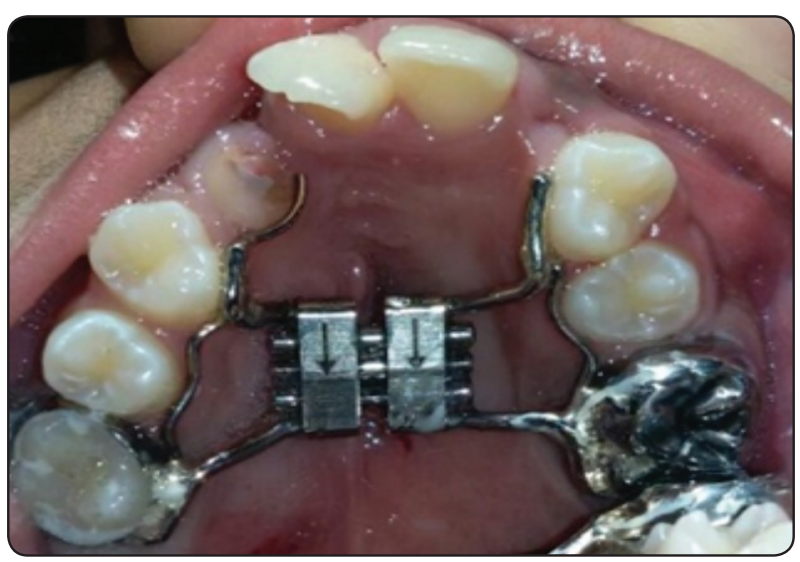

Fig. (1) Occlusal view of the banded fan-shaped expander, with extension arms reaching to the most anterior tooth in crossbite.

immediately after removal of the expander (this image was also used as the pre- alveolar cleft graft surgery). For image acquisition, participants were standing with their chin on chin support, wearing a vacuum retainer on their lower teeth to ensure separation of the occlusion, with the Frankfort horizontal plane parallel to the floor. They were asked to hold their breath after the end of expiration, without swallowing. This position is stable and has high reproducibility for measurement. The CBCT scans were performed at $90 \mathrm{kV}, 14 \mathrm{~mA}$, scan time of 20 seconds, with a single 360 rotation and 400 $\mu \mathrm{m}$ voxel size. All tomographic scans were done using the Planmeca ProMax* scanner and data for each subject was reconstructed by the Planmeca Romexis** imaging software and stored in (DICOM) format.

Measurements were analyzed at T1 and T2 for transverse changes in the anterior and posterior regions of the maxilla. Posterior maxillary measurements were taken at the level of permanent $1^{\text {st }}$ molar while the anterior measurements were taken at the level of the most anterior teeth

\footnotetext{
* PLANMECA, ProMax, 3D Classic, Helsinki, Finland.

** Planmeca Romexis viewer (version 4.4.2.R).

*** Anatomage, Sanjose, CA, USA.
}

supporting the appliance. CBCT images that were stored in DICOM format files were processed into volumetric images by using InVivo 5 version 5.2 software $^{* * *}$. A craniofacial customized analysis was created for this study. Transverse skeletal change was assessed linearly by measuring the interjugale distance IJ, Figure (3). Alveolar expansion was evaluated by calculating the buccal maxillary width BMW. Fig. (4). (20) Amount of dental tipping was measured through the dental tipping angle DTA $^{(19)}$ Fig. (5), in addition to the linear measurement inter crown distance ICD which was measured posteriorly at the central fossa of the right upper first permanent molar to the left first permanent molar, and anteriorly at the mid-point between mesial and distal fossa of the deciduous canine or center of the central fossa of first deciduous molar or first premolar bilaterally. ${ }^{(12)}$ The Inter furcation distance IFD was assigned at the first molar posteriorly and inter-cementoenamel junction ICEJ measured at the most anterior tooth supporting the appliance. Fig (6). Mandibular dental decompensation was evaluated through mandibular inter- crown distance Mnd IC posteriorly and anteriorly. Fig (7).

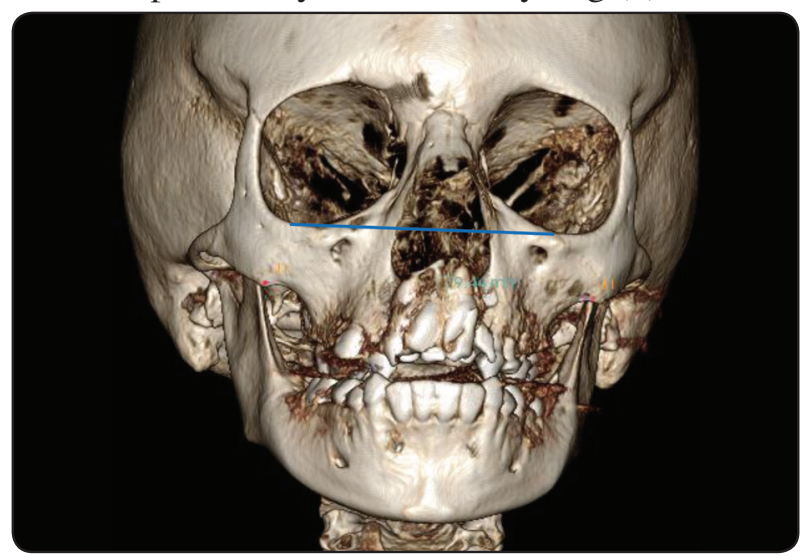

Fig. (3) The distance from the most superior point of the concavity of the maxillary bone as it joins the zygomatic process on the right to the same point on the patient's left (Jugale right-JR to Jugle left-JL). 


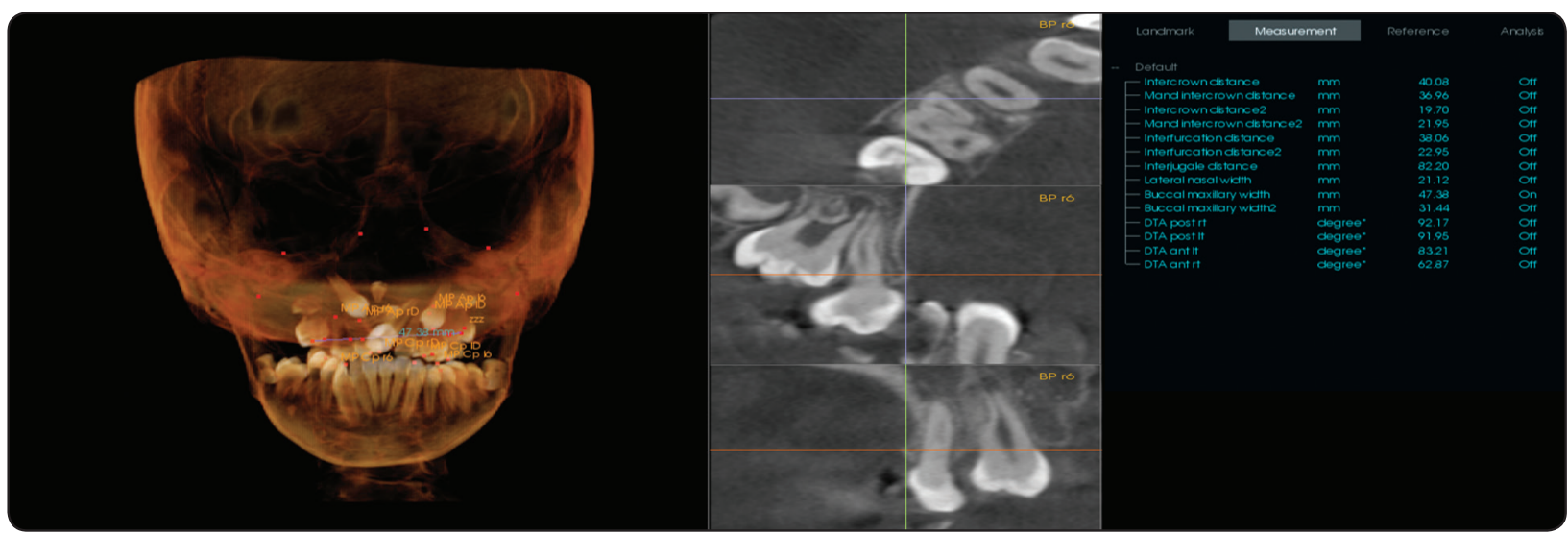

Fig. (4) BMW was measured at two points; the most lateral point on the buccal alveolar plate opposing to the trifurcation of the first permanent molar bilaterally to assess the posterior width, and from the most lateral point on the labial alveolar plate at the level of the CEJ of the most anterior tooth included in the appliance (deciduous canine, first primary molar or first premolar) on both sides to determine the change in anterior width.

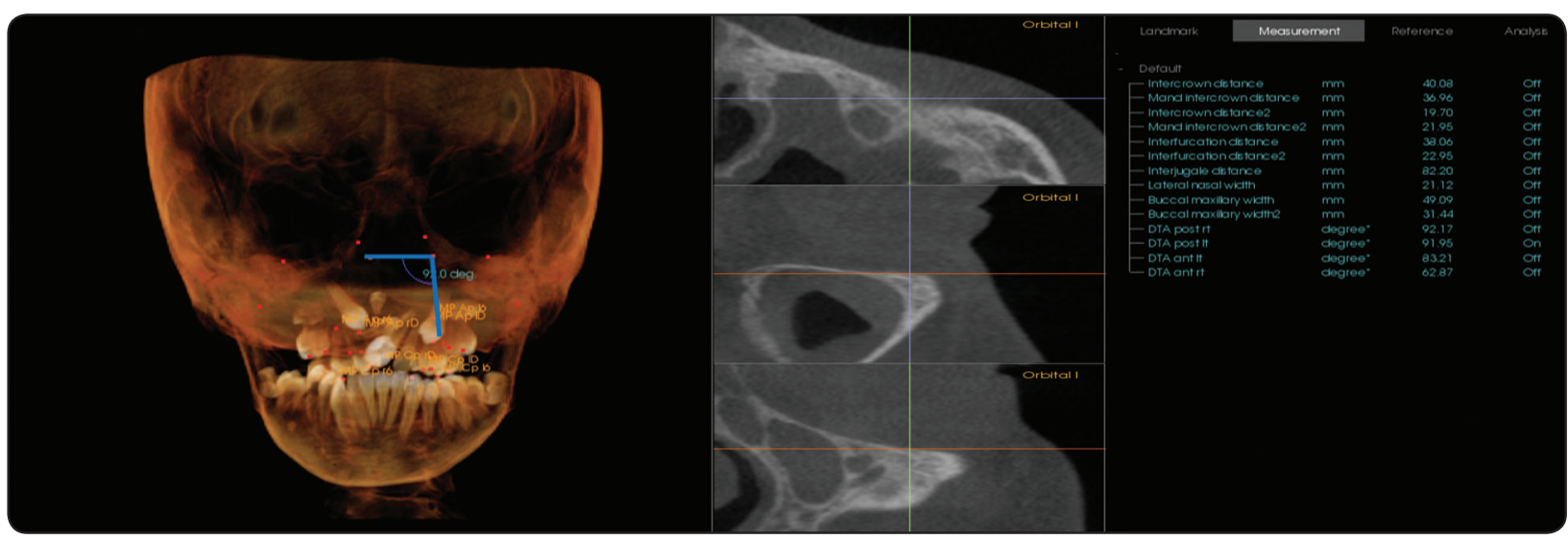

Fig. (5) DT angle measuring dental tipping measured by intersecting Orbital line (line connecting right and left Orbitale) and the permanent first molar long axis (line connecting first molar mesio-palatal cusp and palatal root apex).

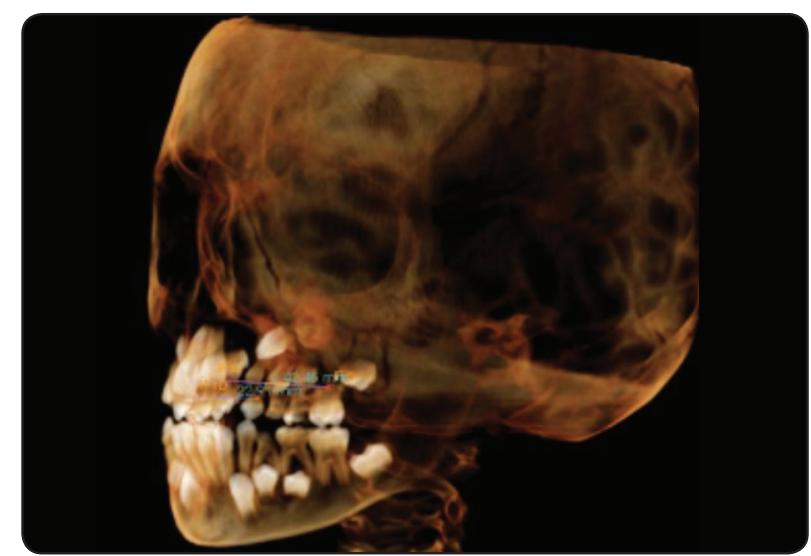

Fig. (6) IFD is the distance between the point of the right and left first molar root trunk before it divides into 3 , and ICEJ is the measurement between the cemento-enamel junction on both deciduous canines. 


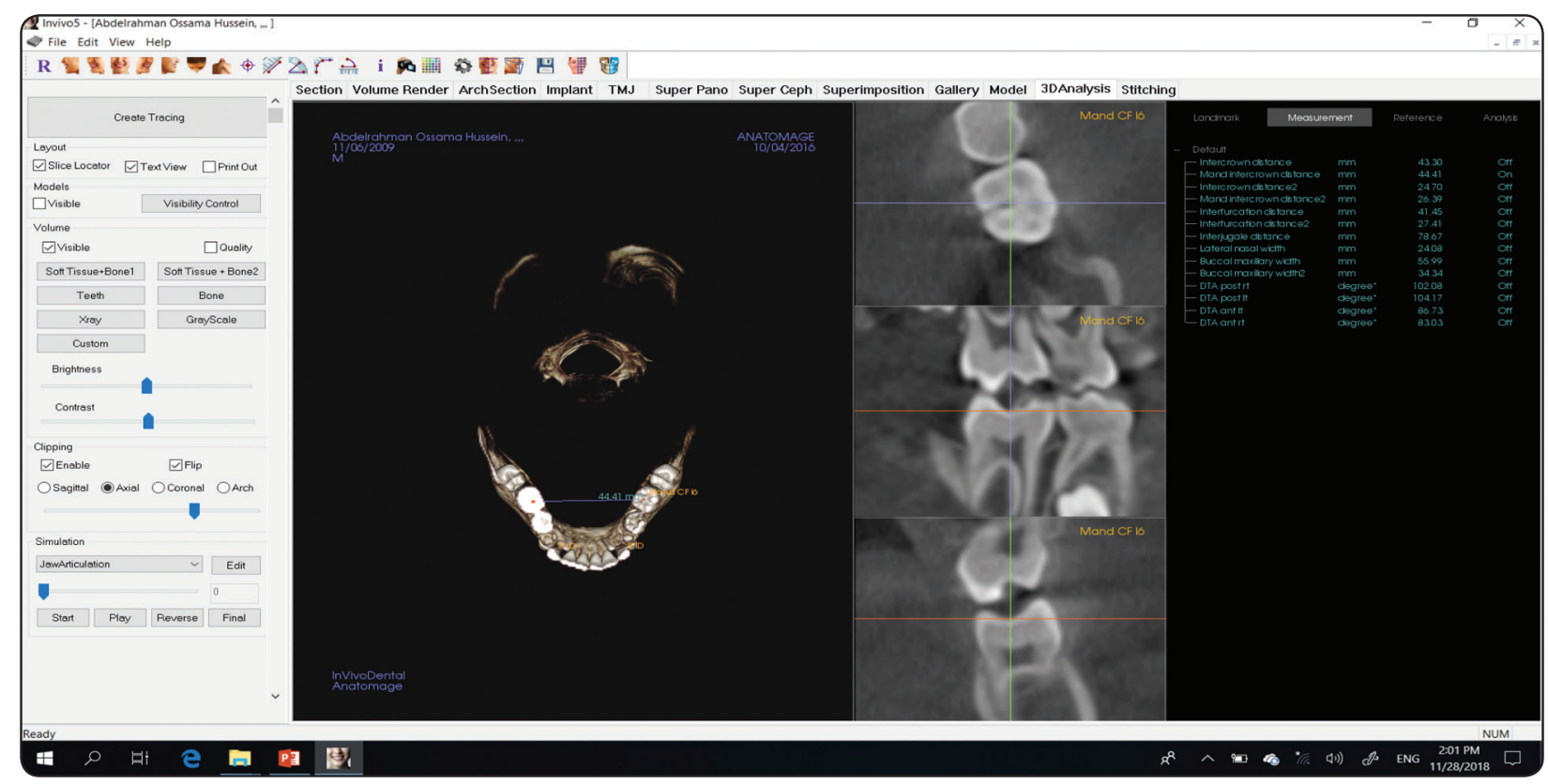

Fig. (7) Mnd IC intermolar width measured at the midpoint of the central fossa of the mandibular first permanent molar, interpremolar width measured at the midpoint of the central fossa of the lower first premolar.

\section{RESULTS}

Statistical analysis was performed by IBM $^{*}$ SPSS $^{* *}$ (version 17). The significance level was considered at $\mathrm{P}<0.05$ by paired $t$ test. KolmogorovSmirnova and Shapiro-Wilk tests of normality were used to test normality hypothesis of all quantitative variables for further choice of appropriate parametric and non-parametric tests. Paired sample $\mathrm{t}$ test was used for testing change within the same group. While independent sample t test was used for comparing the mean changes between the two groups. Dahelberg Error and the Concordance Correlation Coefficient tests were used for the assessment of the reliability of measurements.

A significant skeletal correction of the maxillary constriction was observed for both groups. However, in the Hyrax group, the amount of increase for the basal bone (inter-jugale distance- IJ) was higher than that in the Fan group. For the change in buccal maxillary width (BMW), it was significant for the posterior teeth to be expanded at the alveolar level for the Hyrax group unlike the Fan group, where the increase was non-significant. Anteriorly, there was no statistically difference between the 2 groups, but both groups showed considerable increase. Table 1.

Regarding the dental linear measurements, the results of inter-crown distance (ICD) showed that a statistically significant greater expansion in the anterior region for the fan group rather than the group Hyrax. On the other hand, there was almost no increase in the posterior region for the Fan group unlike the Hyrax group that showed significant expansion posteriorly.

There was no significant difference in mandibular inter- crown expansion (Mnd IC) between the 2 expanders. There were no statistically significant differences in the posterior or anterior regions of the lower dentition. 
TABLE (1) Comparison between change in measurements between both groups; group A (Fan expander) and group B (Hyrax expander)

\begin{tabular}{|c|c|c|c|c|c|c|c|c|c|}
\hline & & & Mean & SD & $\begin{array}{c}\text { Mean } \\
\text { Difference }\end{array}$ & $\begin{array}{l}\text { Std. Error } \\
\text { Difference }\end{array}$ & $\mathbf{t}$ & Probablilty & \\
\hline \multirow{2}{*}{\multicolumn{2}{|c|}{ IJ }} & Fan & 1.54 & 0.62 & \multirow{2}{*}{-1.89} & \multirow{2}{*}{1.34} & \multirow{2}{*}{-1.41} & \multirow{2}{*}{0.18536} & \multirow{2}{*}{$P>0.05$} \\
\hline & & Hyrax & 3.43 & 3.50 & & & & & \\
\hline \multirow{2}{*}{\multicolumn{2}{|c|}{ Post. BMW }} & Fan & 0.86 & 1.76 & \multirow{2}{*}{-3.32} & \multirow{2}{*}{0.88} & \multirow{2}{*}{-3.76} & \multirow{2}{*}{0.00271} & \multirow{2}{*}{$\mathrm{P}<0.01^{*}$} \\
\hline & & Hyrax & 4.18 & 1.53 & & & & & \\
\hline \multirow{2}{*}{\multicolumn{2}{|c|}{ Ant. BMW }} & Fan & 3.87 & 1.48 & \multirow{2}{*}{1.00} & \multirow{2}{*}{0.72} & \multirow{2}{*}{1.38} & \multirow{2}{*}{0.19128} & \multirow{2}{*}{$P>0.05$} \\
\hline & & Hyrax & 2.86 & 1.21 & & & & & \\
\hline \multirow{2}{*}{\multicolumn{2}{|c|}{ Post. ICD }} & Fan & 0.33 & 1.78 & \multirow{2}{*}{-5.78} & \multirow{2}{*}{1.14} & \multirow{2}{*}{-5.08} & \multirow{2}{*}{0.00027} & \multirow{2}{*}{$\mathrm{P}<0.001 *$} \\
\hline & & Hyrax & 6.11 & 2.43 & & & & & \\
\hline \multirow{2}{*}{\multicolumn{2}{|c|}{ Ant. ICD }} & Fan & 7.97 & 2.72 & \multirow{2}{*}{3.79} & \multirow{2}{*}{1.27} & \multirow{2}{*}{2.99} & \multirow{2}{*}{0.01128} & \multirow{2}{*}{$\mathrm{P}<0.05^{*}$} \\
\hline & & Hyrax & 4.18 & 1.96 & & & & & \\
\hline \multirow{8}{*}{ DTA } & Dt Dest & Fan & -0.82 & 6.66 & 1018 & 200 & 251 & 000430 & D $\triangle 001$ * \\
\hline & N. TOst. & Hyrax & 9.36 & 3.82 & -10.10 & 2.90 & - & 0.00450 & $1<0.01$ \\
\hline & It Post & Fan & 2.50 & 12.92 & -302 & 475 & -139 & 000671 & $\mathrm{P}<001 *$ \\
\hline & Ll. $10 \mathrm{Ost}$ & Hyrax & 5.52 & 8.03 & -5.02 & $4.1 \mathrm{~J}$ & - & 0.00071 & $1<0.01$ \\
\hline & $\mathrm{Pt} \Delta \mathrm{nt}$ & Fan & 19.99 & 10.47 & 1233 & 435 & 307 & 000976 & $\mathrm{P}-\Omega 01 *$ \\
\hline & КІ. Аnाt. & Hyrax & 6.66 & 4.76 & (15.55 & 4.53 & 0.01 & 0.00970 & $1<0.01$ \\
\hline & Lt Ant & Fan & 15.76 & 5.36 & 1067 & 364 & 293 & 001257 & $\mathrm{P}<0.05 *$ \\
\hline & Би. . & Hyrax & 5.09 & 8.00 & & & & ונים & \\
\hline & IFD & Fan & 0.13 & 0.88 & 371 & 110 & 338 & 000540 & $\mathrm{P}<001 *$ \\
\hline & HOD & Hyrax & 3.84 & 2.77 & -5.11 & 1.10 & -5.50 & 0.00343 & $1<0.01$ \\
\hline & CEI & Fan & 4.99 & 1.79 & 131 & 0.96 & 136 & 010945 & D) 005 \\
\hline & CEJ & Hyrax & 3.68 & 1.81 & 1.01 & 0.90 & 1.50 & $0.1994 J$ & $1>0.03$ \\
\hline Manc & est ICD & Fan & 0.09 & 0.56 & -033 & 028 & -118 & 026106 & $\mathrm{P}>005$ \\
\hline & & Hyrax & 0.42 & 0.48 & -0.00 & & & 0.20100 & $1-0.00$ \\
\hline Manc & ast ICD & Fan & -0.15 & 0.72 & 010 & 037 & -028 & 078450 & P>005 \\
\hline ivianc & DST. TCD & Hyrax & -0.05 & 0.65 & -0.10 & 0.51 & -0.20 & 0.10450 & $1>0.00$ \\
\hline
\end{tabular}

${ }^{*}$ Significant. Significance level set at $P<0.05$

Inter-furcation distance (IFD) posteriorly was found to be significant statistically as there was more expansion for the Hyrax group, while for the intercomentoenamel junction (ICEJ), the expansion was found to be non-significant for the 2 groups.

Regarding the dental tipping (DTA), buccal inclination of the supporting teeth was found to be statistically significant for both groups especially in the anterior region.

\section{DISCUSSION}

Eversince the introduction of the RPE, orthodontic literature has well documented the skeletal and dentoalveolar effects of the various methodologies of RPE in growing children without oral clefts. ${ }^{(6-}$ 10,26,27) In contrast, there has been scarcity in clinical studies assessing the orthodontic and orthopedic effects of expansion procedures in children with complete CLP especially by means of CBCT. The casual practice of using $\mathrm{CBCT}$ in research has 
raised concerns regarding exposing participants to unnecessary doses of ionizing radiation. However, both American and European guidelines for CBCT rationalize its usage in orthodontic research with clinical benefit to individuals with CLP as one of the indications. ${ }^{(21,26,27)}$

Skeletal correction of the transverse maxillary constriction was achieved by both appliances. However, amount of posterior expansion at the level of the basal bone and the alveolar bone were more in the maxillae expanded by the Hyrax. Anterior alveolar bone width increased significantly in both groups, even though the amount of expansion in the Fan group was higher, there was no statistical difference in the amount achieved between the two groups. This alveolar expansion might be the result of alveolar bending, since the absence of the mid palatal suture contradicts the possibility of true expansion. The unique shape of the Fan expander may be the attribute to the significant alveolar bone expansion.

This design incorporates a posterior hinge that, as mentioned, allows significant expansion anteriorly, but this same design resulted in a restraining effect on the posterior dental dimension. Consequently, amount of dental tipping posteriorly was higher for the hyrax group, and amount of dental tipping in the anterior region was higher for the fan group. In this study, both expanders caused some buccal or labial dental tipping, however, there were major differences in the amount of tipping generated by each expander.

The two expanders established greater anterior tipping than posterior. This could be explained by the increased rigidity of the fan and hyrax expanders obtained by the solder joint to the first permanent molar bands and therefore, as the screw was activated, the bands provided resistance to inclination leading to less tipping posteriorly and more tipping anteriorly as the anterior teeth were only connected by the extended palatal arms.
The degree of tooth translation was assessed by measuring the inter-furcation posteriorly (post. IF) and inter cementoenamel junction anteriorly (ant. CEJ). They were considered points of resistance giving a suggestion whether the teeth moved bodily or by tipping. Accordingly, the amount of expansion increase was much lower than that measured by the ICD. The Hyrax group showed almost even bodily movement both posteriorly and anteriorly, while the Fan group showed bodily expansion anteriorly only. There was no difference between both expanders in the amount of anterior expansion. These measurements were considered due to the incomplete root formation of the newly erupted permanent $1^{\text {st }}$ molar in many of the children treated in this study and the start of root resorption that was observed in the deciduous anterior teeth caused us to believe that to include the posterior and anterior teeth apices would be unreliable.

At the start of the study, there was a preceding expectation that there would be an increase in the mandibular inter-cuspal distance due to dental decompensation following maxillary arch expansion. However, this expectation was not confirmed and might be attributed to the lack of sufficient intercuspation between upper and lower teeth in most of the children participating.

These obtained results might affect the decision of which maxillary expander should be used in cleft children. When both posterior and anterior expansion is required, the hyrax might be the expander of choice, however close monitoring of the expansion is recommended as posterior expansion might be achieved before anterior expansion is complete. If anterior expansion only is required, the fan expander might be the best option.

The use of CBCT for evaluation of these 2 expanders in children with clefts might have great value, enhancing the knowledge and the possibilities concerning their treatment. Long term evaluation is necessary since we assessed the short term effects of RPE. 


\section{CONCLUSION}

The following could be concluded from this study:

1. Both fan and hyrax expanders were effective in transverse skeletal expansion of the maxillary base.

2. The hyrax expander exhibited significant buccal tooth movement (translation) and dental tipping between crowns of anterior and posterior teeth. Whereas the Fan expander showed anterior dental translation and tipping.

3. There was no significant dental transverse increase in the lower arch.

4. The use of CBCT for evaluation of these 2 expanders in children with clefts might have great value, enhancing the knowledge and the possibilities concerning their treatment.

\section{REFERENCES}

1- Mossey P, Shaw W, Munger R, Murray J, Murthy J, Little J. Global oral health inequalities: challenges in the prevention and management of orofacial clefts and potential solutions. Adv Dent Res. 2011 May;23(2):247-58

2- IPDTOC Working Group. Prevalence at birth of cleft lip with or without cleft palate: data from the International Perinatal Database of Typical Oral clefts. Cleft Palate Craniofac J. 2011 Jan;48(1):66-81

3- Silva O, Ramos A, Abdo R. The influence of unilateral cleft lip and palate on maxillary dental arch morphology. Angle Orthod. 1992 Winter;62(4):283-90.

4- Capelozza I, DeAlmeida A, Ursi W. Rapid maxillary expansion in cleft lip and palate patients. J Clin Orthod. 1994 Jan;28(1):34-9.

5- Aizenbud D, Hafer T, Rachmiel A, Figueroa A, Joachims H, Laufer D. A possible ontological complication due to maxillary expansion in a cleft lip and palate patient. Ann Maxillofac Surg. 2015 Jan-Jun; 5(1): 32-36.

6- Weissheimer A, Menezes L, Mezomo M, Dias D, Lima E, Rizzatto S. Immediate effects of rapid maxillary expansion with Haas-type and hyrax-type expanders: a randomized clinical trial. Am J Orthod Dentofacial Orthop. 2011 Sep;140(3):366-76.
7- Garrett B, Caruso J, Rungcharassaeng K, Farrage J, Kim J, Taylor G. Skeletal effects to the maxilla after rapid maxillary expansion assessed with cone-beam computed tomography. Am J Orthod Dentofacial Orthop. 2008 Jul; 134(1):8-9.

8- Lione R, Ballanti F, Franchi L, Baccetti T, Cozza P. Treatment and post treatment skeletal effects of rapid maxillary expansion studied with low-dose computed tomography in growing subjects. Am J Orthod Dentofacial Orthop. 2008 Sep;134(3):389-92.

9- Haas A. Rapid expansion of the maxillary dental arch and nasal cavity by opening the midpalatal suture. Angle Orthod. 1961, April 31(2):73-90.

10- Pan X, Qian Y, Yu J, Wang D, Tang Y, Shen G. Biomechanical effects of rapid palatal expansion on the craniofacial skeleton with cleft palate: a three-dimensional finite element analysis. Cleft Palate Craniofac J. 2007 Mar;44(2):149-54.

11- Figueiredo D, Bartolomeo F, Romualdo C, Palomo J, Horta C, Andrade I, et al. Dentoskeletal effects of 3 maxillary expanders in patients with clefts: A cone-beam computed tomography study. Am J Orthod Dentofacial Orthop. 2014 Jul; 146(1):73-81.

12- Oliveira D, Bartolomeo F, Cardinal L, Figueiredo D, Palomo J, Andrade I. An alternative clinical approach to achieve greater anterior than posterior maxillary expansion in cleft lip and palate patients. J Craniofac Surg. 2014 Nov;25(6):e523-6.

13- Mah J, Yi L, Huang R, Choo H. Advanced applications of cone beam computed tomography in orthodontics. Semin Orthod. 2011; 17: 57-71.

14- Weissheimer A, Menezes L, Mezomo M, Dias D, Lima A, Rizzatto S. Immediate effects of rapid maxillary expansion with Haas-type and hyrax-type expanders. Am J Orthod Dentofacial Orthop 2011;140:366-76.

15- Howe R. Palatal expansion using a bonded appliance. Am J Orthod 1982; 82: 464-8.

16- Hakan E, Palomo J. Three-dimensional evaluation of upper airway following rapid maxillary expansion. A CBCT study. Angle Orthod March 2014;84(2):265-73.

17- Handelman C, Wang L, BeGole E, Haas A. Nonsurgical rapid maxillary expansion in adults: report on 47 cases using the Haas expander. Angle Orthod 2000;70:129-144.

18- Wertz R. Skeletal and dental changes accompanying rapid midpalatal suture opening. Am J Orthod 1970; 58: 41-66. 
19- Garrett B, Caruso J, Rungcharassaeng K, Farrage J, Kim J, Taylor G. Skeletal effects to the maxilla after rapid maxillary expansion assessed with cone-beam computed tomography. Am J Orthod Dentofacial Orthop. 2008; 134: $1-11$.

20- Almeida A, Ozawa T, Alves A, Janson G, Lauris J, Ioshida $\mathrm{M}$, et al. Slow versus rapid maxillary expansion in bilateral cleft lip and palate: a CBCT randomized clinical trial. Clin Oral Invest (2017) 21: 1789-99.

21- Figueiredo D, Cardina L, Bartolomeo F, Palomo J, Horta M, Oliveira D, et al. Effects of rapid maxillary expansion in cleft patients resulting from the use of two different expanders. Dental Press J Orthod. 2016;21(6):82-90.

22- Janson G, Alves A, Garib D, Calil L, Almeida A. Analysis of the dentoalveolar effects of slow and rapid maxillary expansion in complete bilateral cleft lip and palate patients: a randomized clinical trial. Clin Oral Invest. 2016; 20(7):1837-47.

23- Ayub P, Janson G, Gribel B, Lara T, Grib D. Analysis of the maxillary dental arch after rapid maxillary expansion in patients with unilateral complete cleft lip and palate. Am J Orthod Dentofacial Orthop. 2016;149(5):705-15.
24- Corekci B, Goyenc Y. Dentofacial changes from fan-type rapid maxillary expansion vs traditional rapid maxillary expansion in early mixed dentation. Angle Orthod. 2013 Sep;83(5):842-50.

25- Chung C. Font B. Skeletal and dental changes in the sagittal, vertical and transverse dimensions after rapid palatal expansion. Am J Orthod Dentofacial Orthop. 2004; 126: 569-75.

26- European Comission (2011) Radiation protection no 172: cone beam CT for dental and maxillofacial radiology. Evidence based guidelines. A report prepared by the SEDENTEXCT project. Publications office, Luxembourg City 25. Cited from "Almeida A, Ozawa T, Alves A, Janson G, Lauris J, Ioshida M, et al. Slow versus rapid maxillary expansion in bilateral cleft lip and palate: a CBCT randomized clinical trial. Clin Oral Invest (2017) 21: 1789-99."

27- American Academy of Oral and Maxillofacial Radiology (2013) Clinical recommendations regarding use of cone beam computed tomography in orthodontics. [corrected]. Position statement by the American Academy of oral and maxillofacial radiology. Oral Surg Oral Med Oral Pathol Oral Radiol 116:238-257. 